\title{
Multiple Organ Dysfunction Caused by a Ruptured Aortic Sinus Aneurysm: A Case Report
}

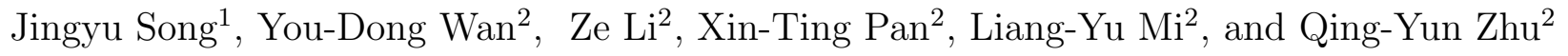 \\ ${ }^{1}$ The Affiliated Hospital of Qingdao University \\ ${ }^{2}$ Affiliation not available
}

April 28, 2020

\begin{abstract}
Background: Aortic sinus aneurysms are congenital malformations that include left, right, and non-coronary sinus aneurysms. Rupture of non-coronary sinus aneurysms is rare; however, the scope of complications may not be fully understood due to the rarity of this situation. This report presents a rare case of multiple organ dysfunction caused by a ruptured non-coronary sinus aneurysm. To the best of our knowledge, no relevant report has been published on aortic sinus aneurysms that lead to multiple organ dysfunction. Case report: A 26-year-old woman presented with cough, sputum, nausea, and vomiting over three days. Computed tomography revealed thickening of the right lung texture and right pleural effusion. No obvious abnormality was found on cardiac ultrasound. After admission, the patient's symptoms worsened, and her heart, liver, and kidney functions gradually deteriorated. After a physical examination and cardiac ultrasound examination, it was discovered that the aorta had a non-coronary sinus rupture that entered the right atrium. On the basis of these findings, we surgically repaired the aortic sinus, resulting in the patient's dramatic recovery. The heart, liver, and kidney functions dramatically improved. Conclusion: A ruptured aortic sinus aneurysm can induce heart, liver, and kidney dysfunction or even failure, and this multi-organ dysfunction can be alleviated by repairing the ruptured aortic sinus aneurysm. After a ruptured aortic sinus aneurysm has been identified, surgery should be performed as soon as possible to prevent further complications.
\end{abstract}

Multiple Organ Dysfunction Caused by a Ruptured Aortic Sinus Aneurysm: A Case Report

Jing-Yu Song, You-Dong Wan\#, Ze Li, Xin-Ting Pan*, Liang-Yu Mi, Qing-Yun Zhu

Department of Emergency Intensive Care Unit, the Affiliated Hospital of Qingdao University, Qingdao 266000,China

Corresponding author: Xin-Ting Pan, Email:0536pxt@163.com

\section{Abstract}

Background:Aortic sinus aneurysms are congenital malformations that include left, right, and non-coronary sinus aneurysms. Rupture of non-coronary sinus aneurysms is rare; however, the scope of complications may not be fully understood due to the rarity of this situation. This report presents a rare case of multiple organ dysfunction caused by a ruptured non-coronary sinus aneurysm. To the best of our knowledge, no relevant report has been published on aortic sinus aneurysms that lead to multiple organ dysfunction.

Case report: A 26-year-old woman presented with cough, sputum, nausea, and vomiting over three days. Computed tomography revealed thickening of the right lung texture and right pleural effusion. No obvious abnormality was found on cardiac ultrasound. After admission, the patient's symptoms worsened, and her heart, liver, and kidney functions gradually deteriorated. After a physical examination and cardiac ultrasound examination, it was discovered that the aorta had a non-coronary sinus rupture that entered the 
right atrium. On the basis of these findings, we surgically repaired the aortic sinus, resulting in the patient's dramatic recovery. The heart, liver, and kidney functions dramatically improved.

Conclusion: A ruptured aortic sinus aneurysm can induce heart, liver, and kidney dysfunction or even failure, and this multi-organ dysfunction can be alleviated by repairing the ruptured aortic sinus aneurysm. After a ruptured aortic sinus aneurysm has been identified, surgery should be performed as soon as possible to prevent further complications.

MeSH Keywords :Congenital diseases, Multiple organ dysfunction, Rupture of aortic sinus aneurysm

\section{Background:}

Aneurysms of the sinus of Valsalva are rare and most cases are congenital. It is generally suspected that the wall of the sinus of Valsalva lacks normal elastic fibers and media; thus, continuous arterial pressure leads to the progressive development of the aneurysm, which can eventually lead to a rupture [1-3]. Aortic sinus aneurysms are rare and can be classified as either congenital or acquired. Aortic sinus aneurysms have a higher incidence rate in males than in females, and in individuals aged 20 to 40 years old [4]. Most aortic sinus aneurysms originate from the right coronary sinus, followed by the non-coronary sinus, and finally the left coronary sinus. Formation of an aortic sinus aneurysm occurs because of the congenital lack of continuity of aortic media and annulus fibrosus, and structural defects in the aortic annulus result in subsequent dysfunction of fibrous tissue connection with the aortic annulus to ultimately form a weak area. The impact of long-term high-velocity blood flow is such that aortic sinus aneurysms gradually develop and finally rupture either because of infection or severe cough, among other reasons [5, 6]. The majority of nonruptured aortic sinus aneurysms give no clinical symptoms and are detected during physical examinations. In the case of a ruptured aneurysm of the aortic sinus, aortic-cardiac shunts may occur, presenting as potential progressive congestive heart failure, acute chest pain with dyspnea in severe cases, or a cardiac arrest in extremely severe cases [7].

Multiple organ dysfunction caused by aortic sinus aneurysms has not been previously reported. Here we present a case of a giant aortic sinus aneurysm rupture into the right atrium that led to multiple organ dysfunction in the heart, liver, and kidneys. This case may serve as a reference for future diagnosis and treatment of similar presentations.

\section{Case Report:}

A 26-year-old woman presented to the hospital with a cough, expectoration, nausea, and vomiting of three days' duration. The patient denied chest tightness or shortness of breath. CT showed right lung texture thickening and right pleural effusion. No obvious abnormality was found on cardiac ultrasound. The patient had self-treated with unspecified traditional Chinese medicine, but symptoms did not improve.

The patient had no history of developmental abnormalities or cardiopulmonary disease evident on previous medical examinations. On admission, physical examination revealed the following: body temperature of 36.5 , heart rate of 107 beats per minute, breathing rate of 21 beats per minute, and blood pressure of 128/59 mmHg. The patient had normal mental status, jugular vein distension, stable breathing, coarse breathing sounds in both lungs, reduced breath sounds in both lower lung fields, regular rhythm of the heart, no obvious pathological murmurs, mild tenderness in the right upper abdomen, rebound pain, and a negative Murphy's sign. The remainder of the physical examination was within normal limits. Laboratory examinations were notable for the following: NT-pro BNP 3,427 pg/mL, ALT $349 \mathrm{U} / \mathrm{L}$, AST $241 \mathrm{U} / \mathrm{L}$, total bilirubin $19.41 \mu \mathrm{mol} / \mathrm{L}$, creatinine $70.4 \mu \mathrm{mol} / \mathrm{L}$, PT(Prothrombin Time ) ratio 1.62, PT percentage $52 \%$, platelets $257 \times 10^{9} / \mathrm{L}$, and lactic acid $1.7 \mathrm{mmol} / \mathrm{L}$ (Figure 1 and Table 1 ). The patient received myocardial contractility strengthening therapy, hepatoprotective therapy, anti-infective therapy, and symptomatic treatment.

Two days following treatment, the patient showed symptoms of anxiety, pallor, dry mouth, sweating, slight cyanosis of limbs, and chills. Electrocardiogram (ECG) revealed that her heart rate was 121 beats per minute. Her breathing rate was 25 beats per minute, and blood pressure was $81 / 47 \mathrm{mmHg}$. A physical 
examination indicated that the patient had obvious jugular vein distension, and a systolic murmur of grade $3 / 6$ in the mitral area. In addition, the patient's urine output decreased every hour. Echocardiography was required for further diagnosis. Echocardiography plays an increasingly important role in the diagnosis and postoperative evaluation of ruptured aortic sinus aneurysm; it can clearly and intuitively reveal the location, shape, size, rupture status, and complications of aortic sinus aneurysms, as well as hemodynamic changes $[8,9]$. A cardiac ultrasound revealed that the left atrium, right atrium, and right ventricle were enlarged, while the left ventricle was normal in size. The aorta without coronary sinus bulged into the right atrium, due to a $2.6 \mathrm{~cm} \times 1.9 \mathrm{~cm}$ sinus tumor, thin cystic wall, multiple ruptures, and a groove size of about $0.77 \mathrm{~cm}$, which broke into the right atrium. An additional rupture of about $0.3 \mathrm{~cm}$ near the opening of the coronary sinus was present, which partly flowed into the coronary sinus. A continuous diversion signal from left to right was seen at the breach, with a peak velocity of $4.4 \mathrm{~m} / \mathrm{s}$ and a peak pressure difference of $76 \mathrm{mmHg}$. The myocardial thickness of the ventricular septum and left ventricular free were normal, and mild regurgitation was seen during the mitral period of the mitral and tricuspid valves. A liquid dark area was observed in the left chest cavity, with a depth of about $8.4 \mathrm{~cm}$. These results led us to diagnose the patient with congenital heart disease, rupture of aortic sinus aneurysm (rupture of non-coronary sinus entering into right atrium), aorticright atrial continuous shunt, right atrial enlargement, left ventricular enlargement, tricuspid regurgitation, mitral regurgitation, pericardial effusion, and pleural effusion (Figure 2). The patient's liver function tests were as follows: ALT 4,505 U/L, AST 6,740 U/L, total bilirubin $48.51 \mu \mathrm{mol} / \mathrm{L}$, creatinine $185 \mu \mathrm{mol} / \mathrm{L}, \mathrm{PT}$ ratio 3.64, PT percentage 19\%, platelets $296 \times 10^{9} / \mathrm{L}$, and lactic acid of $11 \mathrm{mmol} / \mathrm{L}$ (Figure 1 and Table 1).

This patient also had deteriorated function of the liver, kidney, and other organs, as well as aggravated peripheral circulatory failure. The patient underwent surgical repair of the aortic sinus and tricuspid valve through direct vision and intraoperative application of a pacemaker under general anesthesia. Immediately, the patient was intraoperatively diagnosed with a ruptured aortic sinus aneurysm. Liver, kidney, and routine blood tests were performed three days after the operation, all of which improved significantly. Two weeks after the operation, there were no obvious abnormalities on cardiac ultrasound, or on tests of liver or kidney function. The patient recovered with out-of-bed activity and was discharged from the hospital to rehabilitation.

\section{Discussion:}

The sinus of Valsalva rarely ruptures due to congenital or acquired causes. Congenital causes are vulnerability or deficiency of the elastic lamina, as in this case [10]. Symptoms of sinus aneurysm rupture depend on the speed and the size of rupture, as well as on the specific heart cavity penetrated. The incidence of right coronary sinus aneurysm rupture is high. A sinus aneurysm can penetrate any cardiac cavity, mainly in the right ventricle and right atrium. Aortic valve sinus aneurysms usually rupture into the right low-pressure heart cavity after rupture, and blood flows from the high-pressure aorta into the low-pressure right ventricular cavity. Because of the substantial pressure gradient, a large number of left to right shunts are generated, and the hemodynamic changes are similar to those of a patent ductus arteriosus. The blood flow of the pulmonary circulation is increased, and the right ventricular load is increased. This causes enlargement of the right ventricle, pulmonary hypertension, and right heart failure. Aortic valve sinus aneurysms rupture into the right atrium; as a result, the pressure in the right atrium increases significantly, the right atrium expands significantly, blood flow back to the upper and lower vena cava is blocked, and symptoms of right heart failure appear. The disease can exist alone or may co-exist with cardiac malformations such as a ventricular septal defect [11-13]

The typical sign of this event is a heart murmur produced after the rupture of the sinus tumor. It generally presents as a continuous two-stage heart murmur between the third and fourth ribs on the left edge of the sternum, and there may be a tremor of grade III or higher. Other possible indicators include peripheral vascular signs such as water pulse or gunshot sound. There may also be signs of right heart failure such as a jugular vein engorgement or hepatomegaly.

Our patient was a young woman with no abnormal medical history. In this case, we analyzed the cause of the disease because the patient's cardiac output increased due to frequent night work and strong physical work, 
and the cardiac load was heavier. A sudden increase in intrathoracic pressure caused by a cough led to the rupture of an aortic non-coronary sinus aneurysm, obstruction of superior and inferior vena cava blood reflux due to rupture of the right atrium, expansion of the right atrium, and possible acute right heart failure. It subsequently developed into systemic circulation congestion, resulting in heart, liver, and kidney dysfunction and even failure. Of these, liver failure was the most obvious symptom, showing continued increase in transaminase levels on the assessment of liver function and total bilirubin, caused by liver congestion, with the highest level of transaminase reaching over 6,000 U/L. A decrease in the production of coagulation factors after liver congestion led to abnormal PT ratio and PT percentage. An increase in portal vein pressure caused by liver congestion resulted in hypersplenism that decreased the platelet count. After repair of the patient's ruptured aortic sinus aneurysm, we observed an alleviation in hepatic congestion and a linear decrease in transaminase, with a slow decrease in total bilirubin. The detection of postoperative blood system indicators suggested improvement in the disease. Renal congestion caused by systemic circulation congestion was characterized by increased creatinine and urea nitrogen levels, which also began to decrease after surgery. Preoperative dynamic arterial blood gas analysis revealed that lactic acid continued to rise, considering the possibility of sympathetic nerve excitation after heart failure. Systemic vasospasm and poor circulation of the blood microcirculation system may have resulted from insufficient tissue perfusion. Meanwhile, the patient underwent gradual postoperative recovery of cardiac function, improved symptoms of ischemia and hypoxia of the microcirculatory system and decreased lactic acid levels. These results validated the fact that the hepatorenal insufficiency was caused by systemic circulation congestion due to rupture of an aortic sinus aneurysm. At present, there are no case reports reduced liver and kidney dysfunction or even failure and blood system disorder resulting from the rupture of an aortic sinus aneurysm. The presentation of this case may provide a basis for the diagnosis and treatment of patients with multiple organ dysfunction as the presenting symptoms with gradual development of symptoms such as dyspnea and fatigue.

\section{Conclusion:}

Based on the present case report, we conclude that: (1) the role of physical examination should be emphasized, and basic clinical skills should not be neglected by attaching importance solely to supplementary examination and laboratory tests; (2) a ruptured aortic sinus aneurysm can induce heart, liver, and kidney dysfunction or even failure; (3) after an aortic sinus aneurysm has ruptured, surgery should be performed as soon as possible. Multiple organ dysfunctions can improved by repairing the aortic sinus aneurysm rupture and eliminating rupture-induced hemodynamic changes. Otherwise, the patient may have a delayed condition of illness and may even result in death in the most severe cases.

\section{Reference:}

1. Ring W S . Congenital Heart Surgery Nomenclature and Database Project: aortic aneurysm, sinus of valsalva aneurysm, and aortic dissection[J]. The Annals of Thoracic Surgery, 2000, 69(3-supp-S1):0-163.

2. Edwards, J. E, Burchell, H. B. The Pathological Anatomy of Deficiencies between the Aortic Root and the Heart, including Aortic Sinus Aneurysms[J]. Thorax, 12(2):125-139.

3. Edward T. D. Hoey, Gurpreet Singh Gulati, Sandeep Singh, et a1. The role of multi-modality imaging for sinus of Valsalva aneurysms[J]. 28(7):1725-1738.

[4] Sinha SK,Khanna NN,Razi M,et a1.Safety and feasibility of transcatheter interruption of ruptured sinus of Valsalva aneurysm using the cocoon duct occluder:immediate resultsandmid-term follow-up[J].Cardiol Res,2017,8(4):154-160.

[5] Troupis J M , Nasis A, Pasricha S , et al. Sinus valsalva aneurysm on cardiac CT angiography: Assessment and detection[J]. Journal of Medical Imaging and Radiation Oncology, 2013, 57(4):444-447.

[6] Jung S H , Yun T J , Im Y M , et al. Ruptured sinus of Valsalva aneurysm: Transaortic repair may cause sinus of Valsalva distortion and aortic regurgitation[J]. J Thorac Cardiovasc Surg, 2008, 135(5):1153-1158.

[7]Bricker A O, Avutu B, Mohammed T L H, et al. Valsalva sinus aneurysms: findings at CT and MR imaging. [J]. Radiographics A Review Publication of the Radiological Society of North America Inc, 2010, 
30(1):99.

[8]CHENG, YANG, XIE, et al. Echocardiographic diagnosis of sinus of Valsalva aneurysm: a 17-year(19952012) experience of 212 surgically treated patients from one single medical center in China [J].International Journal of Cardiology,2014,173(1):33.

[9] Yang Xiangmei, Wang Ming, Zeng Yanli, et al. Significance of Color Dopple Ultrasound in Rupture of Aortic Sinusal Aneurysm and in Its Complications. [J].Chinese J Ultrasound Med. 2008,24 ( 8) : 755.

[10] Takach TJ, Reul GJ, Duncan JM, Cooley DA, Livesay JJ, Ott DA, et al. Sinus of Valsalva aneurysm or fistula: management and outcome. Ann Thorac Surg 1999;68:1573-7.

[11] Tipoo Sultan FA,Basir N,Fatimi S.Aneurysm of sinus of Valsalva.J Coil Physicians Pak,2011,21:173-175.

[12] Sakakibara S, Konno S. Congenital aneurysm of the sinus of Valsalva. Anatomy and classification. Am Heart J 1962;63:405-24.

[13] Masuyama K, Nakamura H, Okamoto N, Tanaka A, Mori N, Nakamura D, et al. Unusual Case of Rupture of right sinus of Valsalva aneurysm into the left ventricle. Circ J 2017;81:577-8.

Figure and Table Legends

Figure 1.Trends in laboratory examination results. Change trends of liver function, kidney function, hemagglutination, platelets and lactate levels during the hospitalization.

Figure 2.Echocardiographyexamination . (A ) The aorta without coronary sinus bulged into the right atrium because of a $2.6 \mathrm{~cm} \times 1.9 \mathrm{~cm}$ sinus tumor, thin cystic wall, multiple ruptures, and a groove size of about $0.77 \mathrm{~cm}$, which broke into the right atrium (arrow). (B ) A continuous diversion signal from left to right was seen at the breach, with a peak velocity of $4.4 \mathrm{~m} / \mathrm{s}$ and a peak pressure difference of $76 \mathrm{mmHg}$, multicolored mosaic turbulence signal to the right atrium (arrow).

Table 1. Comparison of laboratory examination results. The table more intuitively shows the comparison of the liver and kidney function, hemagglutination, platelets, and lactate of the patient two days before the operation, the day of the operation, and the third day after the operation.

\section{Hosted file}

image.docx available at https://authorea.com/users/314934/articles/445272-multiple-organ-dysfunctioncaused-by-a-ruptured-aortic-sinus-aneurysm-a-case-report 